\title{
Comparison of somatostatin receptor expression in patients with neuroendocrine tumours with and without somatostatin analogue treatment imaged with [18F]SiTATE
}

\section{Ralf Eschbach}

University Hospital Munich: Klinikum der Universitat Munchen

\section{Markus Hofmann}

University Hospital Munich: Klinikum der Universitat Munchen

\section{Lukas Späth}

University Hospital Munich: Klinikum der Universitat Munchen

\section{Lena M. Unterrainer}

University Hospital Munich: Klinikum der Universitat Munchen

\section{Gabriel T Sheihk}

University Hospital Munich: Klinikum der Universitat Munchen

\section{Astrid Delker}

University Hospital Munich: Klinikum der Universitat Munchen

\section{Simon Lindner}

University Hospital Munich: Klinikum der Universitat Munchen

\section{Klaus Jurkschat}

Technische Universität Dortmund: Technische Universitat Dortmund

\section{Carmen Wängler}

University Hospital Mannheim of University of Heidelberg Faculty of Medicine Mannheim:

Universitatsklinikum Mannheim

\section{Björn Wängler}

University Hospital Mannheim of University of Heidelberg Faculty of Medicine Mannheim:

Universitatsklinikum Mannheim

\section{Ralf Schirrmacher}

University of Alberta

\section{Reinhold Tiling}

University Hospital Munich: Klinikum der Universitat Munchen

\section{Matthias Brendel}

University Hospital Munich: Klinikum der Universitat Munchen

\section{Vera Wenter}

University Hospital Munich: Klinikum der Universitat Munchen 


\section{Franziska J. Dekorsy}

University Hospital Munich: Klinikum der Universitat Munchen Mathias J Zacherl

University Hospital Munich: Klinikum der Universitat Munchen Andrei Todica

University Hospital Munich: Klinikum der Universitat Munchen

Harun Ilhan

University Hospital Munich: Klinikum der Universitat Munchen

\section{Freba Grawe}

University Hospital Munich: Klinikum der Universitat Munchen

Clemens C. Cyran

University Hospital Munich: Klinikum der Universitat Munchen

\section{Marcus Unterrainer}

University Hospital Munich: Klinikum der Universitat Munchen

\section{Johannes Rübenthaler}

University Hospital Munich: Klinikum der Universitat Munchen

\section{Thomas Knösel}

University Hospital Munich: Klinikum der Universitat Munchen

\section{Tanja Paul}

University Hospital Munich: Klinikum der Universitat Munchen

\section{Stefan Boeck}

University Hospital Munich: Klinikum der Universitat Munchen

\section{Benedikt Westphalen}

University Hospital Munich: Klinikum der Universitat Munchen

\section{Christine Spitzweg}

University Hospital Munich: Klinikum der Universitat Munchen

\section{Christoph J. Auernhammer}

University Hospital Munich: Klinikum der Universitat Munchen

\section{Peter Bartenstein}

University Hospital Munich: Klinikum der Universitat Munchen

\section{Leonie Beyer ( $\sim$ Leonie.Beyer@med.uni-muenchen.de)}

University Hospital Munich: Klinikum der Universitat Munchen https://orcid.org/0000-0002-7358-4495

\section{Research Article}

Keywords: NET, PET/CT, [18F]SiTATE, Somatostatin analogues

Posted Date: February 9th, 2022

DOI: https://doi.org/10.21203/rs.3.rs-1324662/v1 
License: (c) (i) This work is licensed under a Creative Commons Attribution 4.0 International License. Read Full License 


\section{Abstract \\ Purpose}

Somatostatin analogues (SSA) are frequently used in the treatment of neuroendocrine tumours. Recently,

$\left[{ }^{18} \mathrm{~F}\right]$ SiTATE entered the field of somatostatin receptor (SSR) positron emission tomography (PET)/ computed tomography (CT) imaging. The purpose of this study was to compare the SSR-expression of differentiated gastroentero-pancreatic neuroendocrine tumours (GEP-NET) measured by [ $\left.{ }^{18} \mathrm{~F}\right]$ SiTATE$\mathrm{PET} / \mathrm{CT}$ in patients with and without previous treatment with long-acting SSAs to evaluate if SSA treatment needs to be paused prior to $\left[{ }^{18} \mathrm{~F}\right]$ SiTATE-PET/CT.

\section{Methods}

77 patients were examined with standardised $\left[{ }^{18} \mathrm{~F}\right]$ SiTATE-PET/CT within clinical routine: 40 patients with long-acting SSAs up to 28 days prior to PET/CT examination and 37 patients without pre-treatment with SSAs. Maximum and mean standardized uptake values (SUV $V_{\max }$ and $\left.S U V_{\text {mean }}\right)$ of tumours and metastases (liver, lymphnode, mesenteric/peritoneal and bones) as well as representative background tissues (liver, spleen, adrenal gland, blood pool, small intestine, lung, bone) were measured, SUV ratios (SUVR) were calculated between tumours/metastases and liver, likewise between tumours/metastases and corresponding specific background, and compared between the two groups.

\section{Results}

SUV $_{\text {mean }}$ of liver ( $5.4 \pm 1.5$ vs. $\left.6.8 \pm 1.8\right)$ and spleen (17.5 \pm 6.8 vs. $\left.36.7 \pm 10.3\right)$ were significantly lower (p $<0.001)$ and SUV $V_{\text {mean }}$ of blood pool $(1.7 \pm 0.6$ vs. $1.3 \pm 0.3)$ was significantly higher $(p<0.001)$ in patients with SSA pre-treatment compared to patients without. No significant differences between tumour-to-liver and specific tumour-to-background SUVRs were observed between both groups (all $p>$ $0.05)$.

\section{Conclusion}

In patients previously treated with SSAs, a significantly lower SSR expression ([ $\left.{ }^{18} \mathrm{~F}\right] \mathrm{SiTATE}$ uptake) in normal liver and spleen tissue was observed, as previously reported for ${ }^{68} \mathrm{Ga}$-labelled SSAs, without significant reduction of tumour-to-background contrast. Therefore, there is no evidence that SSA treatment needs to be paused prior to $\left[{ }^{18} \mathrm{~F}\right]$ SiTATE-PET/CT.

\section{Introduction}


Overexpression of somatostatin receptors (SSRs) is highly relevant for both diagnostics and therapeutic options in well-differentiated neuroendocrine tumours (NET) [1, 2]. According to the German and European consensus guidelines, treatment with somatostatin analogues (SSAs) is the first line treatment for proliferation control in all well-differentiated metastatic/ non-resectable NET of the Gastro-EnteroPancreatic System (GEP-NET) [3-5]. Diagnostically, ${ }^{68}$ Ga-labeled SSAs are recommended for staging, restaging and therapy monitoring [6, 7]. Therapeutically, ${ }^{177} \mathrm{Lu} / /^{90} \mathrm{Y}$-labeled SSAs are used in later-stage disease in metastatic/ non-resectable GEP-NETs $[4,5]$.

As both therapeutic and diagnostic SSAs bind to SSRs, medication with SSA could potentially reduce the specific binding of the SSA radiotracer in combined positron-emission-tomography/computed tomography (PET/CT) imaging. After treatment with SSA octreotide, in vitro studies indicated internalisation of SSR subtype 2 receptors [8-11]. On the contrary, one study suggested upregulation of SSR expression after incubation of pituitary cells in culture with a SSA [12].

For imaging, a former study revealed an improved visualisation of carcinoid liver metastases by ${ }^{111} \mathrm{In}$ pentetreotide scintigraphy after treatment with cold SSA [13]. The uptake of ${ }^{68} \mathrm{Ga}$-labeled SSAs DOTATATE and DOTATOC in PET/CT imaging was found to be only reduced in the normal organs but not in tumour tissue after SSA medication, leading to an even higher tumour-to-background contrast $[14,15]$. To rule out a potentially reduced binding and impaired therapeutic effect of radioactive SSA for peptide receptor radionuclide therapy (PRRT), medication with long-lasting SSAs needs to be paused at least 30 days and medication with short-acting SSAs for at least 24 hours prior to PRRT [16].

Currently, the first ${ }^{18} \mathrm{~F}$-labelled SSA radioligand, $\left[{ }^{18} \mathrm{~F}\right] \mathrm{SiTATE}$ has been introduced as an alternative to ${ }^{68} \mathrm{Ga}$-labeled SSAs for NET SSR-PET imaging with comparable radiation exposure and promising tumourto-background contrast [17-20]. The aim of this study was to investigate the influence of SSA medication prior to $\left[{ }^{18} \mathrm{~F}\right]$ SITATE PET/CT regarding normal-tissue and tumour uptake of the radiotracer when compared to former ${ }^{68} \mathrm{Ga}$-labeled SSAs to validate its clinical potential.

\section{Material And Methods}

\section{Patient enrolment}

All patients were referred for imaging by their treating endocrinologists and/-or oncologists between March 2019 and April 2021 and gave written informed consent to undergo [ ${ }^{18}$ F]SiTATE-PET/CT following the regulations of the German Pharmaceuticals Act. The study was performed in compliance with the principles of the Declaration of Helsinki and its subsequent amendments [21], and with the approval of the local ethics committee (approval number 21-0102).

\section{PET/CT imaging}


SiTATE was obtained from ABX, Advanced Biomedical Compounds (Radeberg, Germany) and $\left[{ }^{18} \mathrm{~F}\right]$ SiTATE was synthesized as described previously $[17,18,22]$. All quality control data met the release criteria. $\left[{ }^{18} \mathrm{~F}\right]$ SiTATE-PET/CT scans were acquired at the Department of Nuclear Medicine, LMU Munich on a Siemens Biograph mCT flow (Siemens Healthineers, Erlangen, Germany). After intravenous injection of 3 $\pm 1 \mathrm{MBq} / \mathrm{kgBw}$ (median $232 \pm 36 \mathrm{MBq}$, range 152 to 310 ) of $\left[{ }^{18} \mathrm{~F}\right]$ SiTATE, PET scans were acquired $90 \pm$ 15 min after injection for 15-20 min (in flow mode depending on the body height). Patients were asked to empty the bladder if necessary. In 75/77 patients, contrast-enhanced CT scans with $1.5 \mathrm{~mL}$ of iopromide (Ultravist 300, Bayer Healthcare, Leverkusen, Germany) per kilogram of body weight were performed for anatomic localisation; the remaining two cases received diagnostic CT scan without contrast enhancement. The PET scan was acquired by static emission data with a scan speed of $0.7 \mathrm{~mm} / \mathrm{s}$ for both neck and abdominal region and $0.9 \mathrm{~mm} / \mathrm{s}$ for the lung region in flow mode. With CT scans serving for attenuation correction, PET images were reconstructed with a transaxial $200 \times 200$ matrix using TrueX (including TOF, 2 iterations and 21 subsets, 3D Gauss post-filter of 4-mm full-width-half-maximum).

\section{Image analysis}

Image analysis was performed using a dedicated software package (Hermes Hybrid Viewer, Hermes Medical Solutions, Stockholm, Sweden). Uptake in normal organs and tumour uptake (hottest lesion for

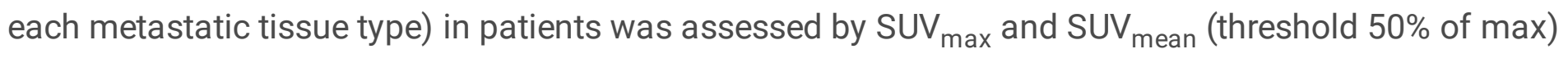
measurements as described previously $[18,20]$. In short, spherical VOls were placed inside the organ parenchyma using a 1-cm diameter VOI for small organs (adrenal glands) and a 2-cm diameter VOI for muscle, liver, spleen, fat tissue, aortic lumen (descending aorta), lung, bone (femur) and small intestine. Tumour-to-liver ratios (TLR) and tumour-to-background ratios (corresponding background for each lesion type, e.g. bone for osseous metastasis) were calculated for all measured tumour lesions according to the clinically relevant Krenning score which has been evaluated for SSTR-PET imaging [23, 24].

\section{Statistical analysis}

Data are reported as mean or median \pm standard deviation or range as stated. Demographics and radiotracer uptake of normal organs (spleen, adrenal gland, liver small intestine, blood-pool, lung, bone) and tumour lesions were compared between group using a student's t-test for metric variables and a Fisher exact/ Chi-square test for contingency analysis of non-metric data. Radiotracer uptake was correlated with the time after SSA-injection using a Pearson's correlation coefficient. GraphPad Prism (version 8.4.3, GraphPad Software Inc., San Diego, CA) was used for statistical analysis and illustration of results. A significance level of $p<0.05$ was applied in all analyses.

\section{Results}

\section{Patients}

All patients tolerated the examination well and did not report any unforeseen symptoms or adverse reactions. No drug-related pharmacologic effects or physiologic responses occurred. Thirty-seven male 
and forty female patients with differentiated NETs and a median age of 63 (range 24 - 86) underwent a $\left[{ }^{18} \mathrm{~F}\right]$ SiTATE-PET/CT. Primary tumour locations included the small intestine (ileum $n=25$, duodenum $n=$ 2, jejunum $n=1$, not specified. $n=7$ ), large intestine (colon $n=2$, appendix $n=2$, rectum $n=1$ ), pancreas $(n=27)$, liver $(n=1)$, stomach $(n=1)$ and primary tumour was not detectable (carcinoma of unknown primary) in $n=8$ patients. Prior to PET/CT, the majority of patients were known to have hepatic $(n=51)$ metastases. Further metastatic sites included lymph nodes $(n=34)$, bone $(n=22)$, lung $(n=2)$ or peritoneal $(n=12)$ lesions. Most of the patients underwent surgery before $(n=45)$ followed by PRRT $(n=$ 25) and chemotherapy $(n=14)$. Detailed patient characteristics are provided in Table 1.

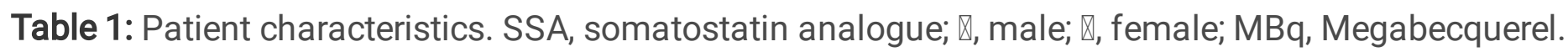

\begin{tabular}{|c|c|c|c|c|}
\hline & All & SSAt & SSA- & $\begin{array}{l}\text { p-value (SSA+vs. } \\
\text { SSA-) }\end{array}$ \\
\hline Sex & $\llbracket 37 \llbracket 40$ & $\varangle 22 \otimes 18$ & $\otimes 15 \bowtie 22$ & 0.256 \\
\hline Age $[y]($ mean \pm SD) & $\begin{array}{l}62.7 \pm \\
12.1\end{array}$ & $\begin{array}{l}63.7 \pm \\
10.2\end{array}$ & $\begin{array}{l}61.6 \pm \\
13.9\end{array}$ & 0.455 \\
\hline $\begin{array}{l}\text { Time since initial diagnosis [m] } \\
(\text { mean } \pm \mathrm{SD})\end{array}$ & $\begin{array}{l}62.3 \pm \\
57.6\end{array}$ & $\begin{array}{l}75.3 \pm \\
49.5\end{array}$ & $\begin{array}{l}48.3 \pm \\
62.9\end{array}$ & 0.039 \\
\hline Ki-67 (mean \pm SD) $n=70$ & $6.1 \pm 5.3$ & $\begin{array}{ll}5.1 & \pm \\
4.8\end{array}$ & $\begin{array}{ll}7.1 & \pm \\
5.7\end{array}$ & 0.127 \\
\hline $\begin{array}{l}\text { Tumour-grading (G1/G2/G3) } \\
\mathrm{n}=73\end{array}$ & $(25 / 47 / 1)$ & $(16 / 23)$ & $(9 / 24 / 1)$ & 0.266 \\
\hline $\mathrm{MBq}($ mean $\pm \mathrm{SD})$ & $233 \pm 36$ & $240 \pm 38$ & $226 \pm 32$ & 0.069 \\
\hline Creatinin $[\mathrm{mg} / \mathrm{dl}]($ mean \pm SD) & $\begin{array}{l}0.94 \pm \\
0.26\end{array}$ & $\begin{array}{l}0.92 \pm \\
0.20\end{array}$ & $\begin{array}{l}0.96 \pm \\
0.32\end{array}$ & 0.612 \\
\hline
\end{tabular}

\section{Biodistribution}

In line with previous studies, the radiotracer uptake in the normal organs was highest in the spleen, followed by the adrenal glands and the liver. Patients undergoing a SSA treatment showed a significantly reduced radiotracer uptake in the spleen $\left(S_{U V} V_{\text {mean }} 17.5\right.$ vs. $\left.36.7, p<0.001\right)$ and the liver $\left(S_{\text {SUV }}\right.$ mean 5.4 vs. $6.8, \mathrm{p}<0.001$ ) when compared to patients without SSA treatment. On the other hand, the radiotracer uptake was significantly higher in the blood pool of patients with ongoing SSA treatment (SUV mean 1.7 vs. 1.3, $p<0.001$ ). For details of the biodistribution in normal organs see Table 2 and Figure 1.

Table 2: Biodistribution of $\left[{ }^{18} \mathrm{~F}\right]$ SiTATE in normal organs. SUV, standard uptake value. 


\begin{tabular}{|lllll|}
\hline SUV $_{\text {mean }}($ mean \pm SD $)$ & All & SSA & SSA- & p-value (SSA+ vs. SSA-) \\
\hline Spleen & $26.8 \pm 12.9$ & $17.5 \pm 6.8$ & $36.7 \pm 10.3$ & $<0.001$ \\
\hline Adrenal gland & $13.5 \pm 5.2$ & $13.2 \pm 5.7$ & $13.7 \pm 4.7$ & 0.700 \\
\hline Liver & $6.1 \pm 1.8$ & $5.4 \pm 1.5$ & $6.8 \pm 1.8$ & $<0.001$ \\
\hline Small intestine & $4.6 \pm 1.3$ & $4.6 \pm 1.4$ & $4.6 \pm 1.3$ & 0.991 \\
\hline Blood pool & $1.5 \pm 0.5$ & $1.7 \pm 0.6$ & $1.3 \pm 0.3$ & $<0.001$ \\
Lung & $0.4 \pm 0.1$ & $0.4 \pm 0.1$ & $0.3 \pm 0.1$ & 0.098 \\
Bone & $0.7 \pm 0.2$ & $0.7 \pm 0.2$ & $0.7 \pm 0.2$ & 0.400 \\
\hline
\end{tabular}

\section{Tumour uptake and tumour-to-background ratios}

Overall, the radiotracer uptake $\left(S U V_{\text {max }}\right)$ to primary or metastatic tumour lesions was not significantly different between patients with/ without ongoing SSA treatment. Also, tumour-to-liver and tumour-tospecific-background ratios did not significantly differ between groups. For details of both groups see Table 3 and Figure 2.

Table 3: Radiotracer uptake of [18F]SiTATE in metastatic tumour lesions. SUV, standard uptake value. 


\begin{tabular}{|c|c|c|c|}
\hline & SSA+ & SSA- & p-value (SSA+ vs. SSA-) \\
\hline \multicolumn{4}{|l|}{ Tumour uptake $\left(S U V_{\text {max }}\right)$} \\
\hline Hottest lesion ( $n=77$ ) & $\begin{array}{l}43.5 \pm \\
32.0\end{array}$ & $\begin{array}{l}42.3 \pm \\
36.9\end{array}$ & 0.969 \\
\hline Pancreas $(n=33)$ & $\begin{array}{l}30.9 \pm \\
46.0\end{array}$ & $\begin{array}{l}43.8 \pm \\
34.0\end{array}$ & 0.414 \\
\hline Bowel $(n=8)$ & $\begin{array}{l}24.4 \pm \\
15.7\end{array}$ & $15.4 \pm 1.6$ & 0.466 \\
\hline Lymph node $(\mathrm{n}=33)$ & $\begin{array}{l}35.8 \pm \\
32.3\end{array}$ & $\begin{array}{l}20.3 \pm \\
13.2\end{array}$ & 0.154 \\
\hline Liver $(n=50)$ & $\begin{array}{l}31.2 \pm \\
14.5\end{array}$ & $\begin{array}{l}39.3 \pm \\
27.2\end{array}$ & 0.180 \\
\hline Lung $(n=3)$ & $\begin{array}{l}16.5 \pm \\
11.1\end{array}$ & & \\
\hline Bone $(n=26)$ & $\begin{array}{l}26.3 \pm \\
40.2\end{array}$ & $15.1 \pm 9.3$ & 0.374 \\
\hline Heart $(n=4)$ & $12.1 \pm 6.3$ & 11.7 & 0.959 \\
\hline Soft tissue $(n=1)$ & 7.7 & & \\
\hline Abdominal $(n=23)$ & $\begin{array}{l}33.2 \pm \\
16.3\end{array}$ & $\begin{array}{l}35.8 \pm \\
55.4\end{array}$ & 0.875 \\
\hline Spleen $(n=1)$ & 6.6 & & \\
\hline Adrenal gland $(n=1)$ & 39.0 & & \\
\hline Ovar $(n=1)$ & & 13.0 & \\
\hline \multicolumn{4}{|l|}{ Tumour-to-liver-ratio $\left(S U V_{\max } / S U V_{\text {mean }}\right)$} \\
\hline Liver $(n=50)$ & $6.1 \pm 2.8$ & $7.1 \pm 5.7$ & 0.430 \\
\hline Lymph node (n=33) & $7.1 \pm 6.3$ & $3.4 \pm 2.9$ & 0.094 \\
\hline Bone $(n=26)$ & $4.7 \pm 5.1$ & $2.7 \pm 1.6$ & 0.229 \\
\hline Abdominal $(n=23)$ & $6.4 \pm 3.2$ & $4.9 \pm 6.5$ & 0.477 \\
\hline \multicolumn{4}{|c|}{ Tumour-to-specific-background-ratio (SUV $\left.\mathrm{max}_{\text {max }} / \mathrm{SUV}_{\text {mean }}\right)$} \\
\hline Hepatic metastasis/ Liver $(n=50)$ & $6.1 \pm 2.8$ & $7.1 \pm 5.7$ & 0.430 \\
\hline Lymph node metastasis/ Blood pool (n=33) & $\begin{array}{l}28.1 \pm \\
39.0\end{array}$ & $\begin{array}{l}18.9 \pm \\
14.0\end{array}$ & 0.477 \\
\hline Osseous metastasis/ Bone $(n=26)$ & $\begin{array}{l}40.5 \pm \\
68.8\end{array}$ & $\begin{array}{l}27.1 \pm \\
18.4\end{array}$ & 0.538 \\
\hline
\end{tabular}




\section{Individual radiotracer uptake under SSA treatment}

Previous group-wise comparison suggests a reduced radiotracer uptake in normal organs but comparable tumour-to-background ratios. Figure $\mathbf{3}$ shows two exemplary patient cases that visually match those results.

Altered $\left[{ }^{18} \mathrm{~F}\right] \mathrm{SiTATE}$ uptake was time-dependent. Figure $\mathbf{4}$ shows the inter-individual correlation between liver, spleen and blood-pool SUV $V_{\text {mean }}$ and hottest lesion SUV $V_{\text {max }}$ with the time after SSA injection with significant correlations for the liver and spleen radiotracer uptake $\left(R_{\text {Liver }}=0.363, p_{\text {Liver }}=0.022 ; R_{\text {Spleen }}=\right.$ $\left.0.515, \mathrm{p}_{\text {Spleen }}=0.001\right)$.

\section{Discussion}

In the present study we investigated the influence of a previous treatment with long-acting nonradioactive SSAs on the SSR-expression in patients with GEP-NETs measured by PET/CT with the new radioactive SSA $\left[{ }^{18} \mathrm{~F}\right] \mathrm{SiTATE}$.

Our results with $\left[{ }^{18} \mathrm{~F}\right] \mathrm{SiTATE}$ are in line with previous clinical studies with several SSR radioligands: Haug et al. investigated 105 NET patients with $\left[{ }^{68} \mathrm{Ga}\right] \mathrm{Ga}$-DOTATATE of whom 35 had been pre-treated with longacting octreotide and reported a significantly lower $\left[{ }^{68} \mathrm{Ga}\right] \mathrm{Ga}$-DOTATATE uptake of non-tumorous spleen and liver in patients treated with SSAs [15]. Lodge et al., who prospectively investigated the effect of a pre-dose of octreotide prior to injection of the radiotracer on the distribution of $\left[{ }^{68} \mathrm{Ga}\right] \mathrm{Ga}$-DOTATOC in 7 patients with GEP-NETs intra-individually within $7 \pm 9$ days observed a decreased radiotracer uptake in normal liver by $25 \%$ and spleen by $47 \%$ with octreotide pre-therapy [14]. Another prospective study of Aalbersberg et al. intra-individually evaluated 34 patients with metastatic NET before and after lantreotide therapy with the result of significantly decreased uptake of non-tumorous liver and spleen [25]. This is in accordance with our results with significant lower $\left[{ }^{18} \mathrm{~F}\right]$ SiTATE background uptake $\left(\mathrm{SUV}_{\text {mean }}\right)$ in liver and spleen tissue in patients with previous SSA treatment, compared to patients without.

Moreover, our results with $\left[{ }^{18} \mathrm{~F}\right]$ SiTATE showed a significantly higher blood pool signal in SSA treated patients. This could reflect a decreased binding and therefore higher number of circulating radioligands because of two potential explanations: a lower SSR density as a consequence of predominantly internalized SSR 2 in neoplastic and physiologic target tissues or SSR saturation with non-radioactive SSAs under therapy [26].

In contrast to Haug et al., who used $\left[{ }^{68} \mathrm{Ga}\right] \mathrm{Ga}$-DOTATATE, in our study with $\left[{ }^{18} \mathrm{~F}\right] \mathrm{SiTATE}$ the radioligand uptake of the non-tumorous background in liver and spleen as well as in blood pool showed a time dependency on the interval since SSA treatment with significant positive correlations between the number 
of days after treatment and radiotracer uptake of liver and spleen, and a trend towards an inverse correlation with the blood pool. This time dependency on the interval since SSA treatment may be explained by the fact that the non-radioactive SSAs compete with the SSR radioligands for receptor binding sites and the quantity of SSR internalization is dependent on the octreotide dose [26]. This means that the more non-radioactive SSAs are circulating in the blood, the more receptors are blocked and internalized, but with reversibility of the internalization over time [27].

Aalbersberg et al. observed an increased tumour uptake leading to an increased tumour-to-liver ratio [25]. Former studies using conventional scintigraphy for the detection of NET metastases before and during treatment with octreotide showed an intra-individually improved visualization of carcinoid liver metastases in 5 midgut NET patients after SSA treatment measured by ${ }^{111}$ In-pentreotide [13] and a higher tumour-to-background ratio in 8 NET patients by using ${ }^{111}$ In-octreotide scintigraphy [28]. In contrast, analogously to Haug et al. and Lodge et al. [14, 15], our results reveal no significant differences in SSR expression and target-to-background-ratios. With $\left[{ }^{18} \mathrm{~F}\right]$ SiTATE there were no significant differences in SUV $_{\text {max }}$ in tumours/ metastastic lesions between the two groups as well as no significant differences in tumour-to-liver and tumour-to-specific-background ratios. Our findings may be partially explained by the fast and efficient internalization of SSR 2 after agonist stimulation under octreotide therapy in vivo in neoplastic as well as in physiologic SSR 2 target tissues [26, 27]. Furthermore, an agonist-induced upregulation of SSR subtypes, which causes an increase of the receptor density in the tumours and metastases of SSA treated patients [12] with a consecutively higher $\left[{ }^{18} \mathrm{~F}\right] \mathrm{SiTATE}$ uptake may be partially masked by SSA occupied receptors [15]. Thus, there might be a steady state of concomitant receptor internalization and overexpression. In long-acting SSAs, the initial pharmacokinetic profile after injection differs between lanreotide depot and octreotide depot formulations [29], while subsequent serum concentrations remainquite stable over $28 \mathrm{~d}$ with both formulations [29].

It has to be mentioned that the above cited scintigraphical studies are limited by several aspects: first, only a very low number of patients was investigated. Secondly, the quantifiability in conventional scintigraphy is reduced compared to PET/CT with SUV calculations. Thirdly, interpretation of these intraindividual scintigraphy results is limited by a possible tumour progression during the 12 month treatment course which may result in an increase of the uptake values.

However, all these study results consistently indicate that octreotide treatment may influence the binding and change the biodistribution of SSR radioligands, but suggest that the diagnostic reliability of somatostatin receptor imaging in NET metastases is not significantly compromised by any previous or concomitant octreotide therapy [13]. Moreover, these findings even underline that SSA treatment may facilitate the detection of NET metastases, mainly driven by a decline in background binding in the liver and spleen rather than an increase in tumour binding, possibly providing an improved tumour delineation [15].

Because of the heterogeneity of NETs and consequently the possibility of various biologic behaviours, it remains uncertain if these findings can be generalized to other types of NET [15]. The presented results 
are promising, but require further investigation in future clinical trials to validate our data intraindividually and also to evaluate the influence of long-acting SSA pre-treatment on radioligand binding in patients that receive PRRT with e.g. $\left[{ }^{177}\right.$ Lu]Lu-DOTATATE.

\section{Conclusions}

A treatment with long-acting SSAs does not reduce the $\left[{ }^{18} \mathrm{~F}\right]$ SiTATE -binding in tumorous target lesions of GEP-NET patients and even reveals a significant lower background signal in non-tumorous liver and spleen tissues, consistently to other radioactive SSA, which could improve demarcation of metastases in these organs. Our results add support to the hypothesis that a previous or concomitant treatment with long-acting SSAs does not unfavourably/adversely influence the SSR expression and therefore confirm the clinical approach not to discontinue/ interrupt any SSA medication prior to a PET/CT examination.

\section{Declarations}

Funding: L.B. was funded by the Munich-Clinician-Scientist-Program (LMU Munich).

Conflicts of interest/Competing interests: C.J.A. has received research contracts (Novartis), lecture honorarium (Ipsen, Novartis, Advanced Accelerator Applications) and honoraria for advisory boards (Advanced Accelerator Applications). H.I. has received research contracts (Novartis). L.B. received honoraria for advisory boards (Bayer, Advanced Accelerator Applications). CBW has received honoraria from Amgen, Bayer, Chugai, Celgene, Falk, GSK, MSD, Merck, Janssen, Ipsen, Roche, Servier, SIRTeX, Taiho; served on advisory boards for Bayer, BMS, Celgene, Servier, Shire/Baxalta, Rafael Pharmaceuticals, RedHill, Roche, has received travel support by Bayer, Celgene, RedHill, Roche, Servier, Taiho and research grants (institutional) by Roche. CBW serves as an officer for European Society of Medical Oncology (ESMO), Deutsche Krebshilfe (DKH), Arbeitsgemeinschaft internistische Onkologie (AIO).

Ethics approval: All procedures performed in studies involving human participants were in accordance with the ethical standards of the institutional and/or national research committee (LMU Munich - approval number 21-0102) and with the 1964 Helsinki declaration and its later amendments.

Consent to participate/ for publication: Written informed consent was obtained from all patients for $\left[{ }^{18}\right.$ F $]$ SiTATE-PET/CT.

Availability of data and material: The data that support the findings of this article are available from the corresponding author (L.B.) upon reasonable request.

Code availability: Not applicable.

\section{References}


1. Reubi J, Waser B, Schaer JC, Laissue JA. Erratum to: Somatostatin receptor sst1-sst5 expression in normal and neoplastic human tissues using receptor autoradiography with subtype-selective ligands. European journal of nuclear medicine. 2001;28:1433. doi:10.1007/s002590100598.

2. Reubi JC, Waser B, Schaer JC, Laissue JA. Somatostatin receptor sst1-sst5 expression in normal and neoplastic human tissues using receptor autoradiography with subtype-selective ligands. European journal of nuclear medicine. 2001;28:836-46. doi:10.1007/s002590100541.

3. AWMF. [Practice guideline neuroendocrine tumors - AWMF-Reg. 021-27]. Zeitschrift fur Gastroenterologie. 2018;56:583-681. doi:10.1055/a-0604-2924.

4. Falconi M, Eriksson B, Kaltsas G, Bartsch DK, Capdevila J, Caplin M, et al. ENETS Consensus Guidelines Update for the Management of Patients with Functional Pancreatic Neuroendocrine Tumors and Non-Functional Pancreatic Neuroendocrine Tumors. Neuroendocrinology. 2016;103:15371. doi: $10.1159 / 000443171$.

5. Garcia-Carbonero R, Sorbye H, Baudin E, Raymond E, Wiedenmann B, Niederle B, et al. ENETS Consensus Guidelines for High-Grade Gastroenteropancreatic Neuroendocrine Tumors and Neuroendocrine Carcinomas. Neuroendocrinology. 2016;103:186-94. doi:10.1159/000443172.

6. Bozkurt MF, Virgolini I, Balogova S, Beheshti M, Rubello D, Decristoforo C, et al. Guideline for PET/CT imaging of neuroendocrine neoplasms with (68)Ga-DOTA-conjugated somatostatin receptor targeting peptides and (18)F-DOPA. European journal of nuclear medicine and molecular imaging. 2017;44:1588-601. doi:10.1007/s00259-017-3728-y.

7. Sundin A, Arnold R, Baudin E, Cwikla JB, Eriksson B, Fanti S, et al. ENETS Consensus Guidelines for the Standards of Care in Neuroendocrine Tumors: Radiological, Nuclear Medicine \& Hybrid Imaging. Neuroendocrinology. 2017;105:212-44. doi:10.1159/000471879.

8. Cescato R, Schulz S, Waser B, Eltschinger V, Rivier JE, Wester HJ, et al. Internalization of sst2, sst3, and sst 5 receptors: effects of somatostatin agonists and antagonists. Journal of nuclear medicine : official publication, Society of Nuclear Medicine. 2006;47:502-11.

9. Ben-Shlomo A, Schmid H, Wawrowsky K, Pichurin O, Hubina E, Chesnokova V, et al. Differential ligand-mediated pituitary somatostatin receptor subtype signaling: implications for corticotroph tumor therapy. The Journal of clinical endocrinology and metabolism. 2009;94:4342-50. doi:10.1210/jc.2009-1311.

10. Arnold R, Trautmann ME, Creutzfeldt W, Benning R, Benning M, Neuhaus C, et al. Somatostatin analogue octreotide and inhibition of tumour growth in metastatic endocrine gastroenteropancreatic tumours. Gut. 1996;38:430-8. doi:10.1136/gut.38.3.430.

11. Lesche S, Lehmann D, Nagel F, Schmid HA, Schulz S. Differential effects of octreotide and pasireotide on somatostatin receptor internalization and trafficking in vitro. The Journal of clinical endocrinology and metabolism. 2009;94:654-61. doi:10.1210/jc.2008-1919.

12. Bruno JF, Xu Y, Berelowitz M. Somatostatin regulates somatostatin receptor subtype mRNA expression in GH3 cells. Biochemical and biophysical research communications. 1994;202:1738-43. doi:10.1006/bbrc.1994.2136. 
13. Dörr U, Räth U, Sautter-Bihl ML, Guzman G, Bach D, Adrian HJ, et al. Improved visualization of carcinoid liver metastases by indium-111 pentetreotide scintigraphy following treatment with cold somatostatin analogue. European journal of nuclear medicine. 1993;20:431-3.

doi:10.1007/bf00209003.

14. Lodge MA, Solnes LB, Chaudhry MA, Wahl RL. Prospective Within-Patient Assessment of the Impact of an Unlabeled Octreotide Pre-dose on the Biodistribution and Tumor Uptake of (68)Ga DOTATOC as Assessed by Dynamic Whole-body PET in Patients with Neuroendocrine Tumors: Implications for Diagnosis and Therapy. Molecular imaging and biology. 2021;23:766-74. doi:10.1007/s11307-02101600-5.

15. Haug AR, Rominger A, Mustafa M, Auernhammer C, Göke B, Schmidt GP, et al. Treatment with octreotide does not reduce tumor uptake of (68)Ga-DOTATATE as measured by PET/CT in patients with neuroendocrine tumors. Journal of nuclear medicine : official publication, Society of Nuclear Medicine. 2011;52:1679-83. doi:10.2967/jnumed.111.089276.

16. Strosberg J, El-Haddad G, Wolin E, Hendifar A, Yao J, Chasen B, et al. Phase 3 Trial of (177)LuDotatate for Midgut Neuroendocrine Tumors. The New England journal of medicine. 2017;376:12535. doi:10.1056/NEJMoa1607427.

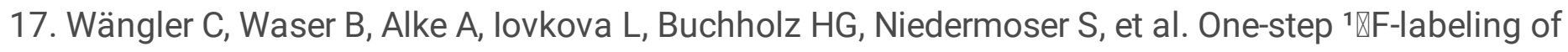
carbohydrate-conjugated octreotate-derivatives containing a silicon-fluoride-acceptor (SiFA): in vitro and in vivo evaluation as tumor imaging agents for positron emission tomography (PET). Bioconjugate chemistry. 2010;21:2289-96. doi:10.1021/bc100316c.

18. Beyer L, Gosewisch A, Lindner S, Völter F, Mittlmeier LM, Tiling R, et al. Dosimetry and optimal scan time of [(18)F]SiTATE-PET/CT in patients with neuroendocrine tumours. European journal of nuclear medicine and molecular imaging. 2021. doi:10.1007/s00259-021-05351-x.

19. Ilhan H, Todica A, Lindner S, Boening G, Gosewisch A, Wängler C, et al. First-in-human (18)F-SiFAlinTATE PET/CT for NET imaging and theranostics. European journal of nuclear medicine and molecular imaging. 2019;46:2400-1. doi:10.1007/s00259-019-04448-8.

20. Ilhan H, Lindner S, Todica A, Cyran CC, Tiling R, Auernhammer CJ, et al. Biodistribution and first clinical results of (18)F-SiFAlin-TATE PET: a novel (18)F-labeled somatostatin analog for imaging of neuroendocrine tumors. European journal of nuclear medicine and molecular imaging. 2020;47:87080. doi:10.1007/s00259-019-04501-6.

21. World Medical Association Declaration of Helsinki: ethical principles for medical research involving human subjects. Journal international de bioethique = International journal of bioethics. 2004;15:124-9.

22. Schirrmacher R, Bradtmöller G, Schirrmacher E, Thews O, Tillmanns J, Siessmeier T, et al. 18Flabeling of peptides by means of an organosilicon-based fluoride acceptor. Angewandte Chemie (International ed in English). 2006;45:6047-50. doi:10.1002/anie.200600795.

23. Hope TA, Calais J, Zhang L, Dieckmann W, Millo C. (111)In-Pentetreotide Scintigraphy Versus (68)GaDOTATATE PET: Impact on Krenning Scores and Effect of Tumor Burden. Journal of nuclear 
medicine : official publication, Society of Nuclear Medicine. 2019;60:1266-9. doi:10.2967/jnumed.118.223016.

24. Krenning EP, Valkema R, Kooij PP, Breeman WA, Bakker WH, deHerder WW, et al. Scintigraphy and radionuclide therapy with [indium-111-labelled-diethyl triamine penta-acetic acid-D-Phe1]-octreotide. Italian journal of gastroenterology and hepatology. 1999;31 Suppl 2:S219-23.

25. Aalbersberg EA, de Wit-van der Veen BJ, Versleijen MWJ, Saveur LJ, Valk GD, Tesselaar MET, et al. Influence of lanreotide on uptake of (68)Ga-DOTATATE in patients with neuroendocrine tumours: a prospective intra-patient evaluation. European journal of nuclear medicine and molecular imaging. 2019;46:696-703. doi:10.1007/s00259-018-4117-x.

26. Reubi JC, Waser B, Cescato R, Gloor B, Stettler C, Christ E. Internalized somatostatin receptor subtype 2 in neuroendocrine tumors of octreotide-treated patients. The Journal of clinical endocrinology and metabolism. 2010;95:2343-50. doi:10.1210/jc.2009-2487.

27. Waser B, Tamma ML, Cescato R, Maecke HR, Reubi JC. Highly efficient in vivo agonist-induced internalization of sst2 receptors in somatostatin target tissues. Journal of nuclear medicine : official publication, Society of Nuclear Medicine. 2009;50:936-41. doi:10.2967/jnumed.108.061457.

28. Janson ET, Kälkner KM, Eriksson B, Westlin JE, Oberg K. Somatostatin receptor scintigraphy during treatment with lanreotide in patients with neuroendocrine tumors. Nuclear medicine and biology. 1999;26:877-82. doi:10.1016/s0969-8051(99)00059-1.

29. Astruc B, Marbach P, Bouterfa H, Denot C, Safari M, Vitaliti A, et al. Long-acting octreotide and prolonged-release lanreotide formulations have different pharmacokinetic profiles. Journal of clinical pharmacology. 2005;45:836-44. doi:10.1177/0091270005277936.

\section{Figures}




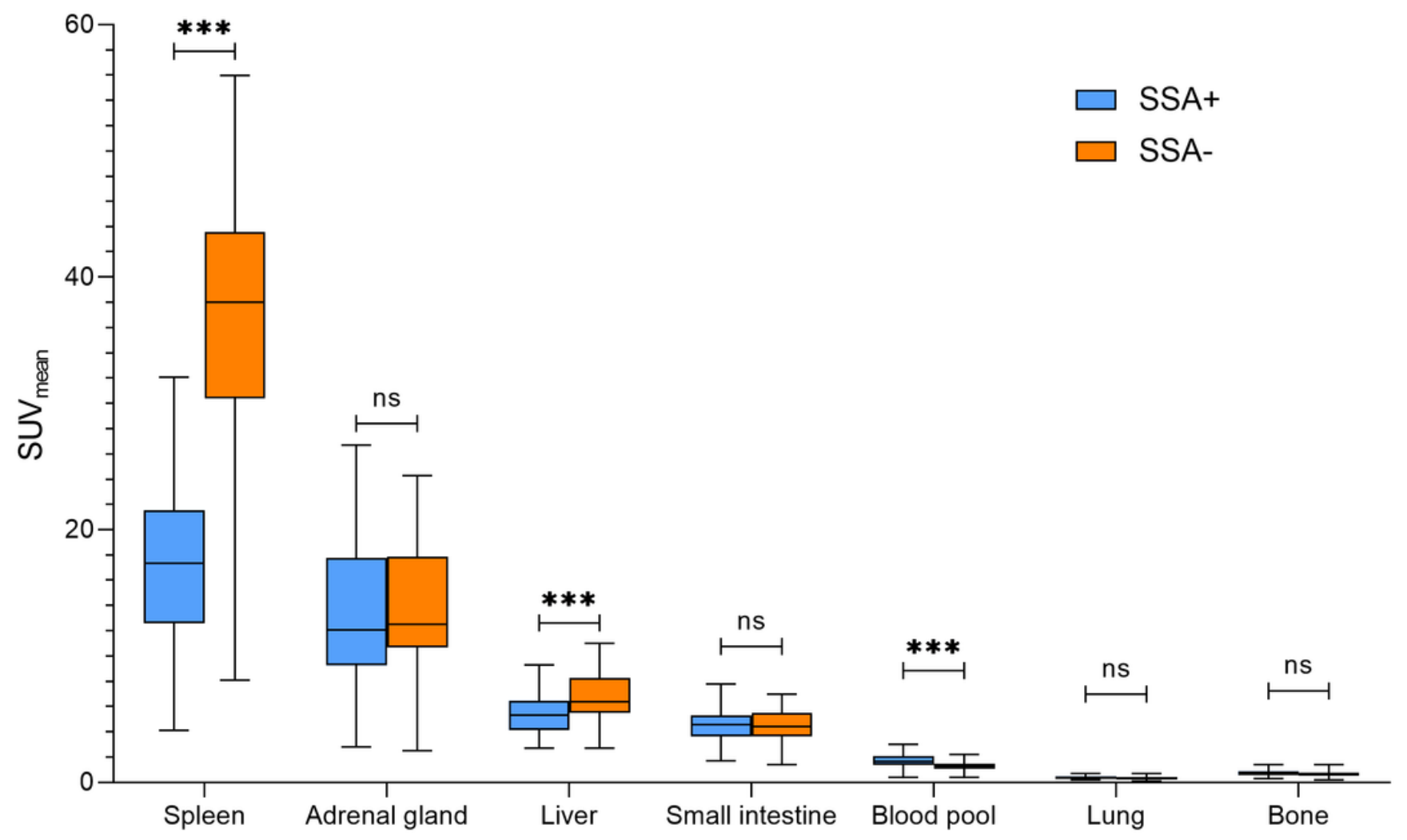

Figure 1

Biodistribution of $\left[{ }^{18} \mathrm{~F}\right] \mathrm{SiTATE}$ in normal organs in patients with (left boxplot)/ without (corresponding right boxplot) SSA treatment. SUV, standard uptake value; SSA, somatostatin analogue. ${ }^{\star \star \star} p<0.001$, ns $=p$-value non-significant. 

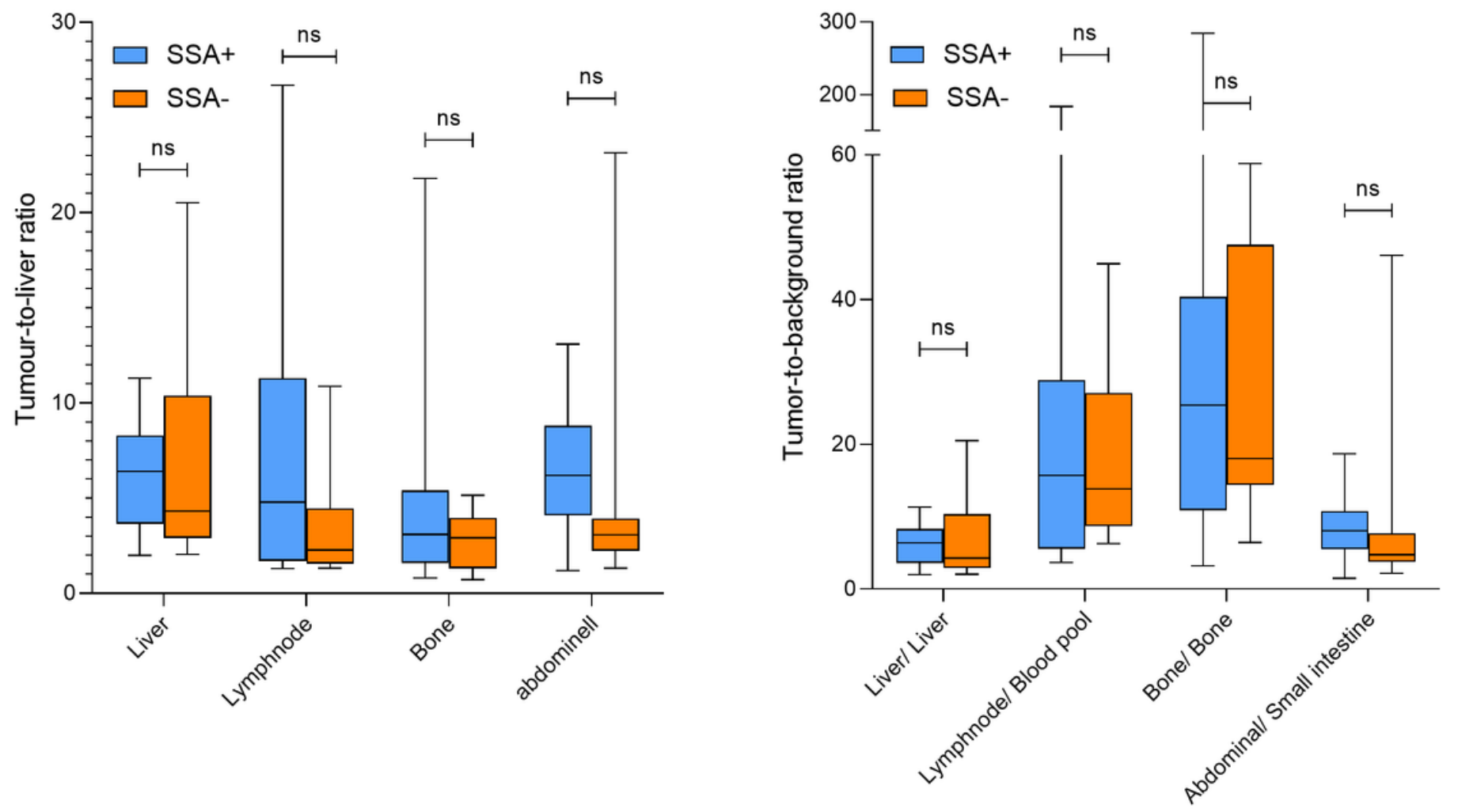

Figure 2

Radiotracer uptake of $\left[{ }^{18} \mathrm{~F}\right] \mathrm{SiTATE}$ in metastatic tumour lesions displayed as tumour-to-liver and tumourto-specific background ratios for patients with (left boxplot)/ without (corresponding right boxplot) SSA treatment. SUV, standard uptake value; SSA, somatostatin analogue; ns = p-value non-significant.
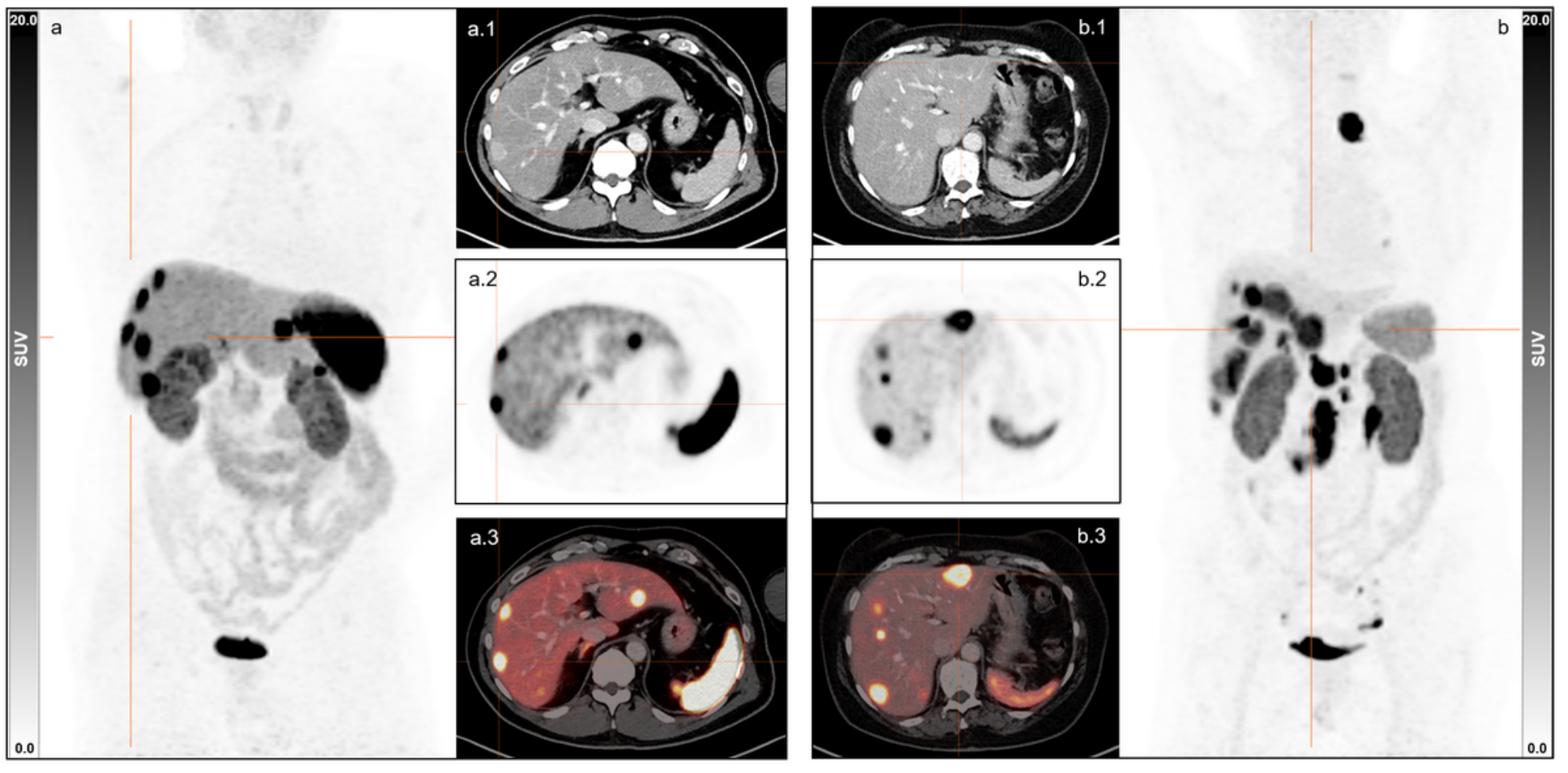


\section{Figure 3}

Exemplary maximum intensity projections images and axial sections (1 CT, 2 PET, 3 fused PET/CT) from patients a without SSA treatment (male, 59 y, 81 kg, 245 MBq, 89 min p.i., creatinine 1.0 mg/dl, liver $S_{\text {SUV }}$ mean 8.3, spleen SUV mean $_{28.0, \text { blood pool SUV }}$ mean 1.3) and $\mathbf{b}$ undergoing SSA treatment (female, 71 y, 64 kg, 281 MBq, 93 min p.i., creatinine 0.7 mg/dl, liver SUV mean 4.1, spleen SUV mean 10.9, blood pool SUV $_{\text {mean }}$ 1.6). SUV, standard uptake value; SSA, somatostatin analogue; MBq, Megabecquerel.
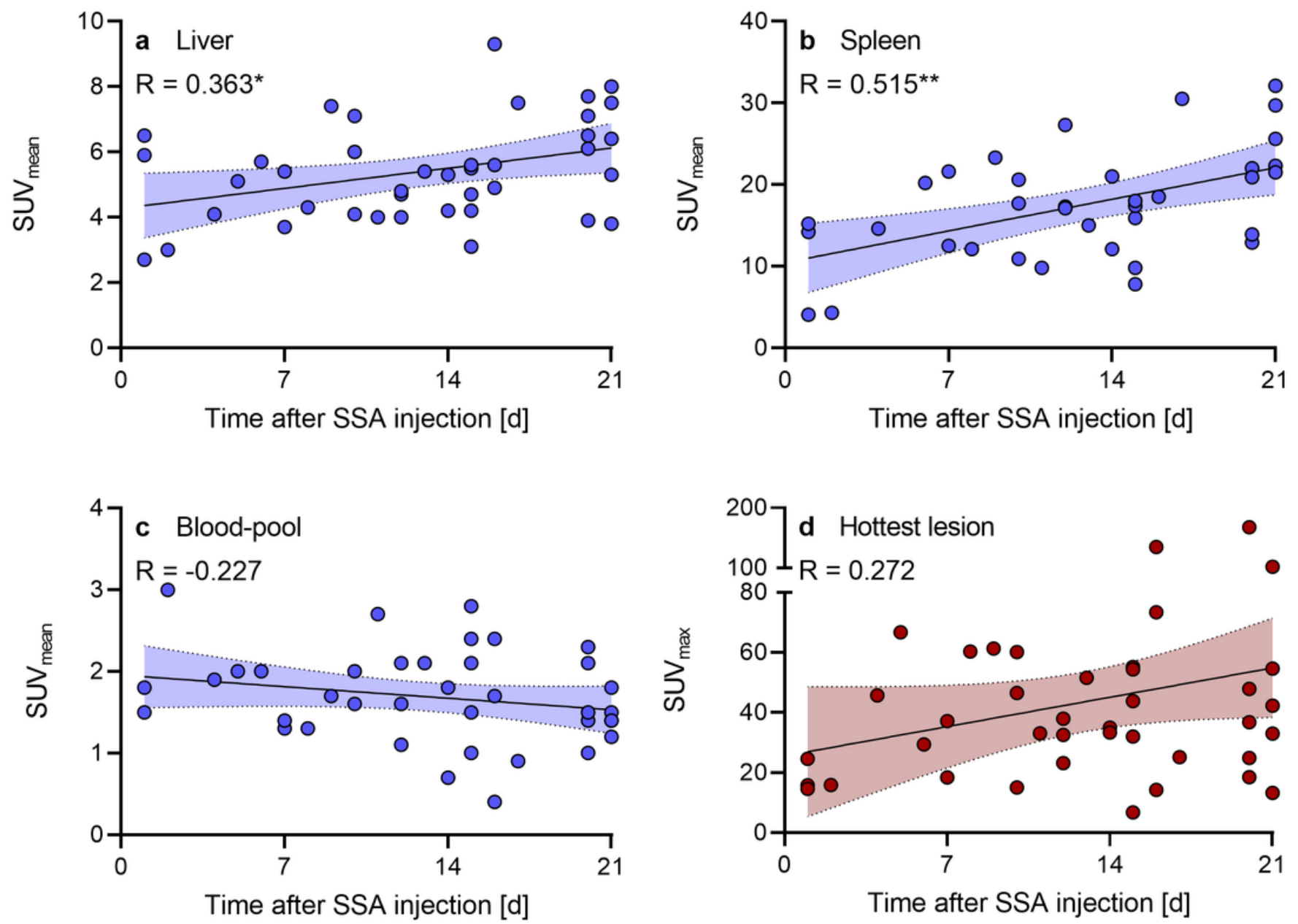

\section{Figure 4}

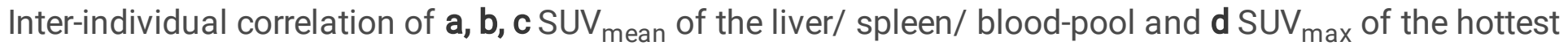
lesion uptake with the time after SSA injection. SUV, standard uptake value; SSA, somatostatin analogue. 